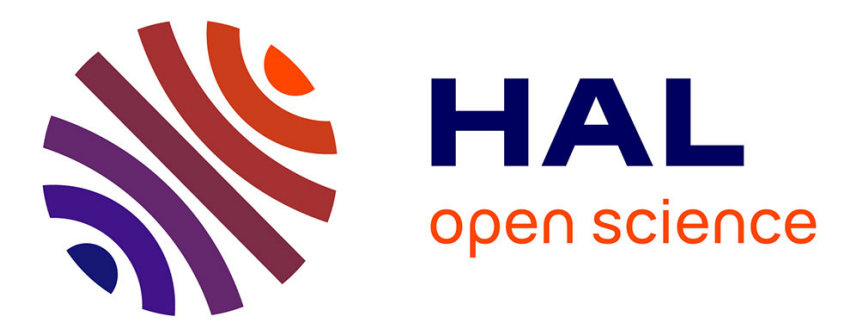

\title{
Quelques données sur les occupations du Clos du Poujol (Aveyron)
}

\author{
Marc Boboeuf, Anne Bridault
}

\section{To cite this version:}

Marc Boboeuf, Anne Bridault. Quelques données sur les occupations du Clos du Poujol (Aveyron). Bulletin de la Société préhistorique française, 1997, 94 (1), pp.51-60. 10.3406/bspf.1997.10633 . hal01545439

\author{
HAL Id: hal-01545439 \\ https://hal.science/hal-01545439
}

Submitted on 31 Oct 2019

HAL is a multi-disciplinary open access archive for the deposit and dissemination of scientific research documents, whether they are published or not. The documents may come from teaching and research institutions in France or abroad, or from public or private research centers.
L'archive ouverte pluridisciplinaire HAL, est destinée au dépôt et à la diffusion de documents scientifiques de niveau recherche, publiés ou non, émanant des établissements d'enseignement et de recherche français ou étrangers, des laboratoires publics ou privés.

\section{(1) $\$(0)$}

Distributed under a Creative Commons Attribution - NonCommercial - ShareAlikel 4.0 


\title{
QUELQUES DONNÉES SUR LES OCCUPATIONS MÉSOLITHIQUES DU CLOS DE POUJOL (AVEYRON)
}

\author{
Marc BOBCEUF et Anne BRIDAULT
}

\section{RÉSUMÉ}

Cet article présente les premiers résultats des recherches archéologiques effectuées au Clos de Poujol (Aveyron, France) en 1990. L'ensemble sédimentaire le plus profond (G) comporte des horizons néolithique ancien et mésolithiques. Les premiers éléments des analyses géoarchéologiques, carpologiques et archéozoologiques sont présentés. $L$ 'ensemble lithique du dépôt $G j$ est étudié en détail et sa signification évaluée. II apparaît que l'analyse des industries définies dans des contextes de dépôts très précis (structures) pourrait apporter des réponses aux questions que pose la variabilité des assemblages lithiques sauveterriens.

\section{ABSTRACT}

Results of archaeological investigations conducted at Clos de Poujol (Aveyron, France) in 1990 are presented in this paper, with an outline of the site potential. The deepest sediments of the recorded archaeological stratigraphy (G) include Early Neolithic and Mesolithic occupation levels. The collected lithic assemblage from the Gj deposit, submitted to detailed analysis, is presented here ; reliable contexts (structures) appear to be highly relevant for the assessment of the variability of Sauveterrean industries. Furthermore, preliminary results of geoarchaeological, archaeobotanical and archaeozoological analyses are presented.

\section{PRÉSENTATION}

Le Clos de Poujol est situé sur le causse de Séverac (commune de Campagnac, fig. 1). La bordure de ce causse, vallonnée et soulignée de falaises, s'apparente à un milieu de moyenne montagne (800-950 m d'altitude). Elle détermine vers le Nord la limite locale d'extension des Grands Causses. Plus au nord, au-delà de la dépression liasique de la Serre $(700 \mathrm{~m})$ et de la vallée permienne du

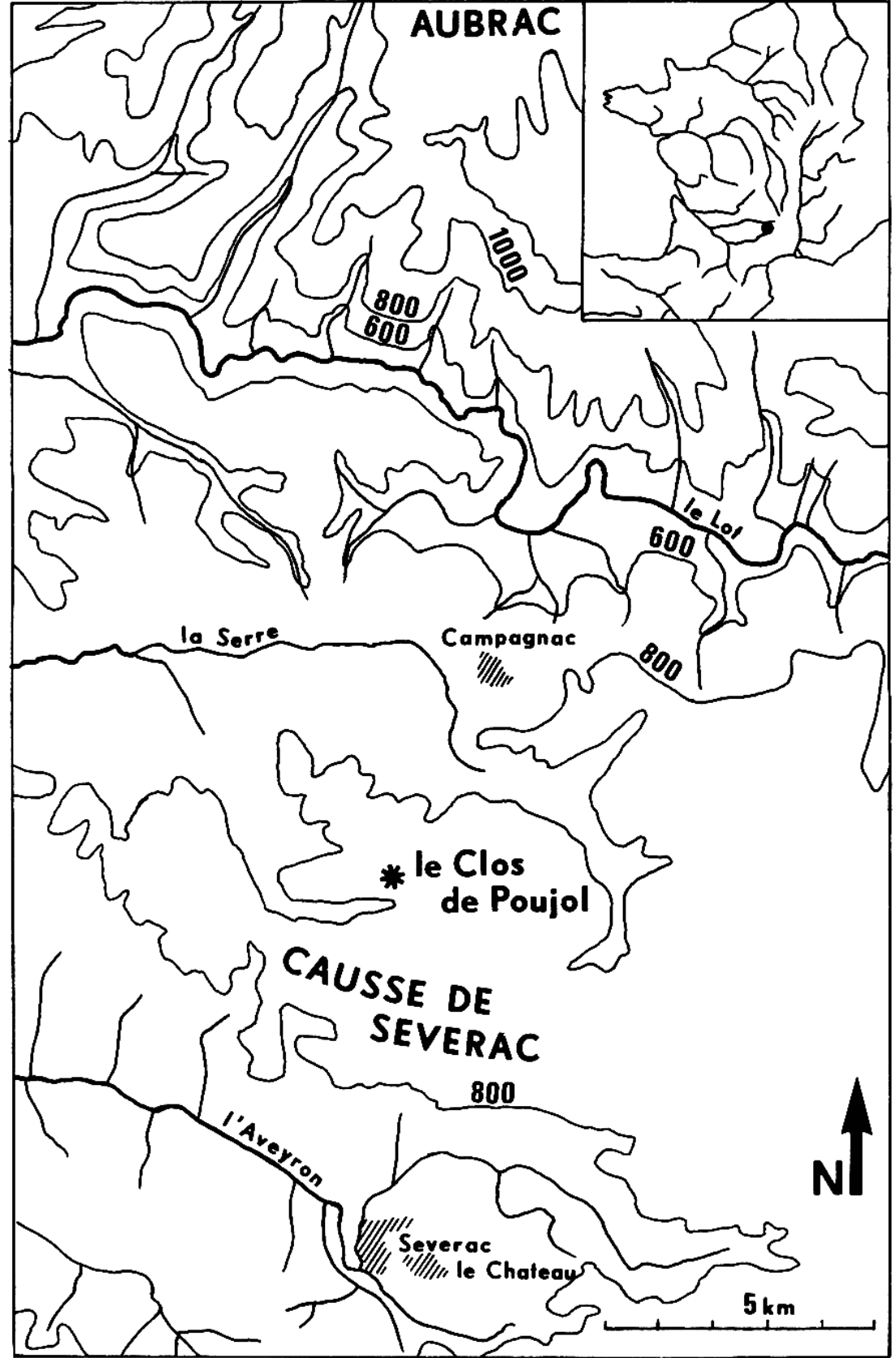

Fig. 1 - Situation topographique du Clos de Poujol.

Lot $(500 \mathrm{~m})$ qui en souligne la séparation, s'élève le haut plateau volcanique de l'Aubrac (1 200-1 $400 \mathrm{~m}$ ).

La baume qui abrite le gisement s'ouvre à 850 mètres d'altitude sur le flanc ouest d'une modeste colline, dans la zone botanique de la série mixte du chêne pubescent et du pin sylvestre des étages subméditérranéens. L'abri est établi au dépend d'une saillie rocheuse, petite barre calcaire rapportée aux séries dolomitiques du Jurassique moyen et supérieur. II s'agit d'une cavité quadrangulaire de 20 mètres carrés dont la voûte, élevée au centre de l'abri à 2 mètres au-dessus du sol actuel, 
est nettement surbaissée sur le pourtour (fig. 2). Le remplissage s'étend vers l'extérieur où se forme une petite terrasse de 15 mètres carrés limitée par la rupture de pente.

\section{HISTORIQUE}

Le gisement fut découvert et fouillé partiellement en 1973 par P.-M. Blanquet. En 1975 un contrôle permit à G.-B. Arnal de reconnaître une stratigraphie composée de quatre couches. Ces travaux sont demeurés inédits (1).

En 1990, une intervention dans le cadre d'une fouille de sauvetage nous était accordée par le Service Régional de l'Archéologie de MidiPyrénées. Ces travaux ont permis l'évaluation partielle du potentiel archéologique encore en place. Nous traiterons ici des premiers résultats issus des travaux de 1990.

\section{ÉLÉMENTS DE STRATIGRAPHIE}

La stratigraphie se compose de deux ensembles principaux. L'ensemble supérieur (ensemble rouge ou $R$ ) est caractérisé par un sédiment brun-rougeâtre assez semblable à celui des dépôts de plein air du causse. Un ensemble sous-jacent (ensemble gris ou G) se distingue par la nette anthropisation de ses dépôts. Au sein de cette couche grise apparaissent les accumulations cendreuses fouillées en 1990. Le substrat rocheux n'a pas été atteint. Nous livrons une description succincte et provisoire de la stratigraphie générale telle qu'elle fut observée seulement en coupe lors des travaux de 1990 et une description plus précise des successions cendreuses fouillées sur le carré F5.

\section{Ensemble rouge ou $\mathbf{R}$}

R1 - Sédiment brun-rougeâtre. Épaisseur variable 5 à $20 \mathrm{~cm}$, possibles surcharges et creusements modernes.

R2 - Sédiment identique et coloré en noir par des charbons de bois nombreux, bien conservés et de grande dimension. Épaisseur $5 \mathrm{~cm}$.

(1) Nous remercions P.-M. Blanquet et G.-B. Arnal pour les facilités accordées dans la consultation et la libre exploitation du produit de leurs fouilles.
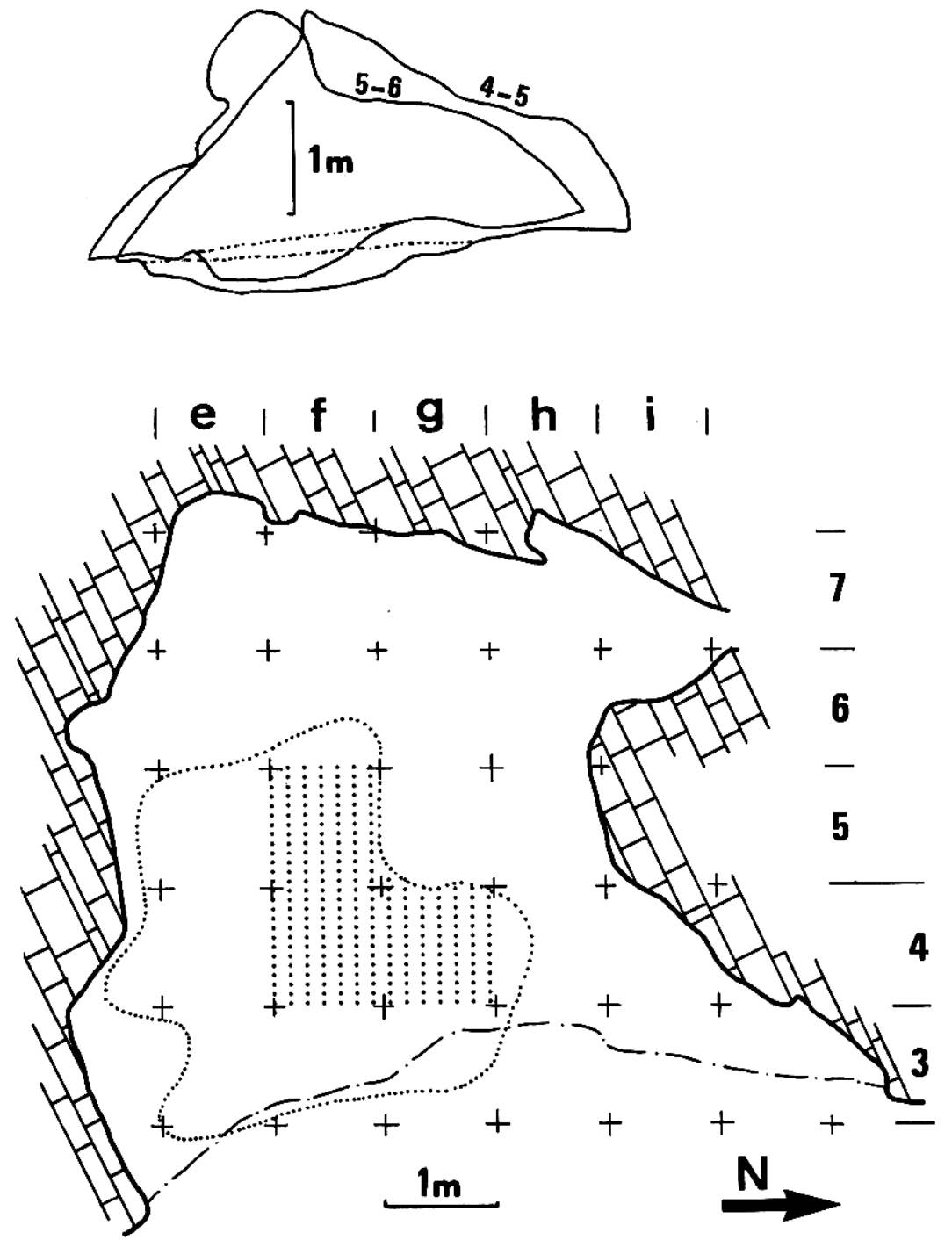

Fig. 2 - Plan de la baume du Clos de Poujol et situation des fouilles. Pointillés : limites des fouilles anciennes. Trame : fouilles de 1990.

R3 - Sédiment brun-rougeâtre. Épaisseur $20 \mathrm{~cm}$.

R4 - Sédiment rouge-grisâtre, dont la nuance de couleur est peutêtre à attribuer à sa situation d'interface entre les ensembles $R$ et $G$. Épaisseur variable, 5 à $10 \mathrm{~cm}$.

\section{Ensemble gris ou G}

G - Sédiment gris. Épaisseur $40 \mathrm{~cm}$.

Sur le carré F5, deux accumulations de sédiment cendreux (Gb1Gf1 et Gj) sont séparées par un horizon de la couche principale $G$,à laquelle elles se substituent localement et donc partiellement (fig. 3). Ces deux accumulations sont à considérer comme des éléments de structure des sols d'occupation de l'ensemble gris $\mathrm{G}$.
Gb1 - Sédiment cendreux compact, blanc, inclusions rares de couleur brune. Épaisseur minimale $10 \mathrm{~cm}$.

Gf1 - Passée de sédiment charbonneux, noir, localisée sous Gb1. Son interface avec $\mathrm{G}$ interstratifiée est rubéfiée. Épaisseur $2 \mathrm{~cm}$.

G interstratifiée - Sédiment gris foncé, induré. Une passée de $1 \mathrm{~cm}$ d'épaisseur, plus sombre avec des nodules roussâtre, est localisée à la base en appui sur Gj. Épaisseur générale $7 \mathrm{~cm}$.

Gj - Sédiment cendreux, jaune pâle. Consistance variable, induré et farineuse. Des passées grisâtres indurées et des feuillets orangées indurées (rubéfaction) sont interstratifiées dans la masse. Ce dépôt est contenu dans une dépression bien marquée (fig. 4). L'interface entre le sédiment jaune et l'encaissant est 

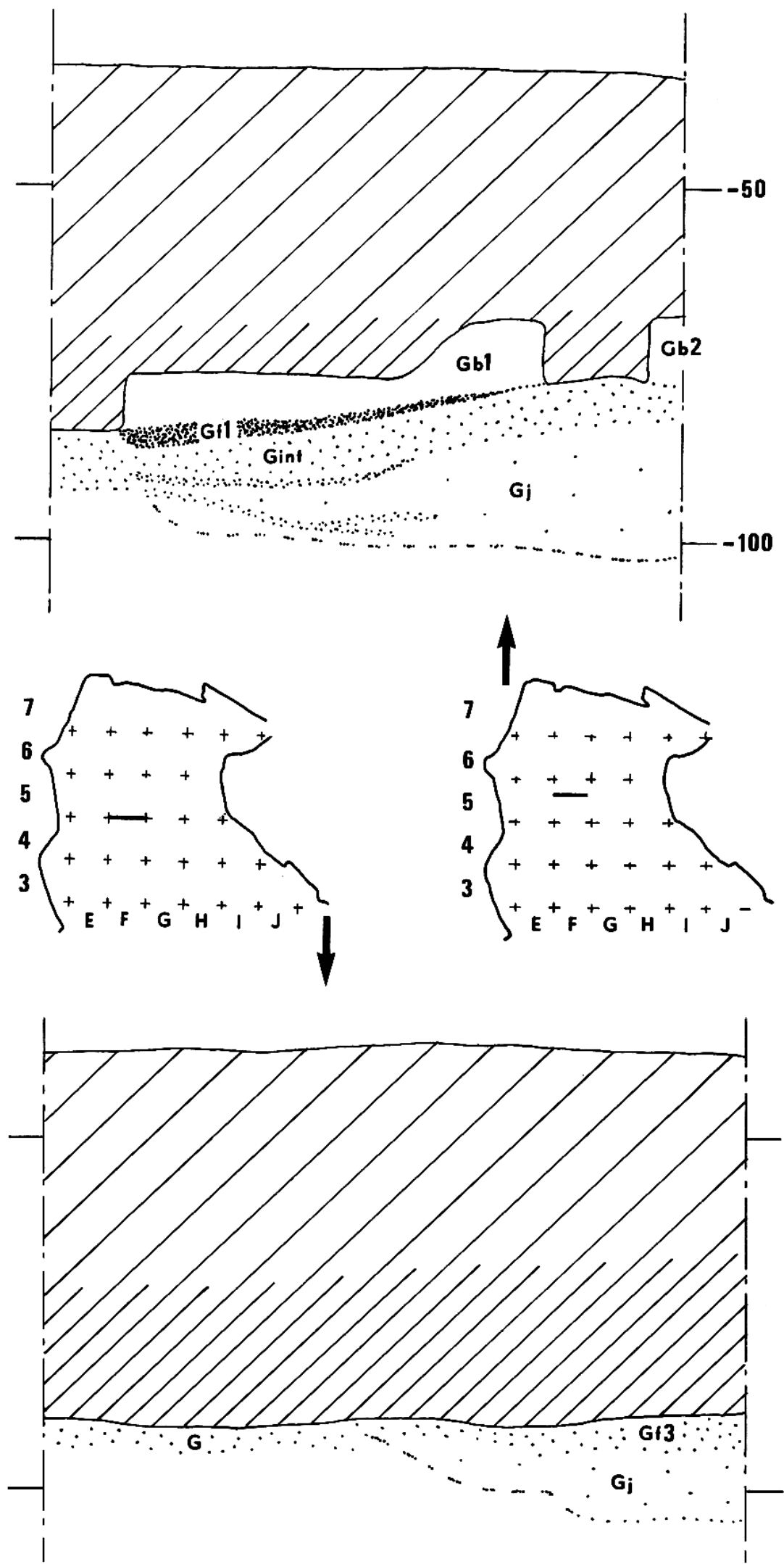

Fig. 3 - Stratigraphie des accumulations cendreuses fouillées sur le carré F5. Hachures : fouilles anciennes. Hachures doubles : déblais et remaniés des fouilles anciennes. Gf3 : langue de $3 \mathrm{~cm}$ d'épaisseur de sédiment gris, interstratifié dans le dépôt Gj au-delà de la coupe 4-5. L'origine de cette "langue", épandage ou débordement en provenance du sol entourant $\mathrm{Gj}$ est à confirmer par les fouilles à venir.

marquée par une fine couche indurée de coloration rouge et orangée (rubéfaction), mouchetée de sédi- ment noir. La forme générale du dépôt s'apparente à une lentille biconvexe d'épaisseur maximale de $20 \mathrm{~cm}$.
Les prélèvements effectués à partir des lambeaux de couche menaçants d'une disparition totale ont permis quelques observations (travaux J.-E. Brochier). L'association entre une cendre très pure (Gb1) et un lit extrêmement riche en microcharbons (Gf1) indique très probablement l'existence d'une aire de combustion en place. Gj est constituée d'un sédiment riche en cendre de bois dans un contexte plus détritique que le foyer Gb1-Gf1 (J.-E. Brochier, in litteris, 1996).

\section{LES DONNÉES}

Pour ce qui concerne les connaissances sur l'environnement naturel au moment de la formation des dépôts, on prendra en compte que les études naturalistes (2) sont à peine engagées et que certains dépôts ont pu contenir des éléments documentaires en relation avec des activités spécifiques.

L'industrie lithique sera traduite dans la typologie de N. Valdeyron (1994) bien adaptée à décrire les industries mésolithiques sauveterriennes. Les descripteurs des produits de débitage seront ceux utilisés par J.-G. Rozoy $(1968,1978)$.

\section{- Dépôts Gb1-Gf1}

Ces deux unités, séparées à la fouille, sont à regrouper et doivent être considérées comme les témoins relictuels d'une aire de combustion en place. Les fouilles anciennes ont emporté le contour de cette accumulation. On ne saura pas s'il s'agissait d'une structure à plat ou en cuvette/fosse.

Les cendres de bois (Gb1) qui composent la partie supérieure de ce foyer indiquent la combustion de bois d'Angiospermes (travaux J.-E. Brochier). Le Noisetier est attesté par un fragment de péricarpe de noisette (détermination P. Marinval).

La faune déterminée (tabl. 3) est représentée par un fragment de bois de cerf.

Deux lambeaux ont été fouillés sur le carré F5. Le principal était une banquette de $60 \mathrm{~cm}$ de longueur sur $15 \mathrm{~cm}$ de largeur où fut observé la

(2) Nous remercions J.-E. Brochier, P. Marinval et $\mathrm{J}$. Cataliotti-Valdina pour les premiers résultats obtenus. 


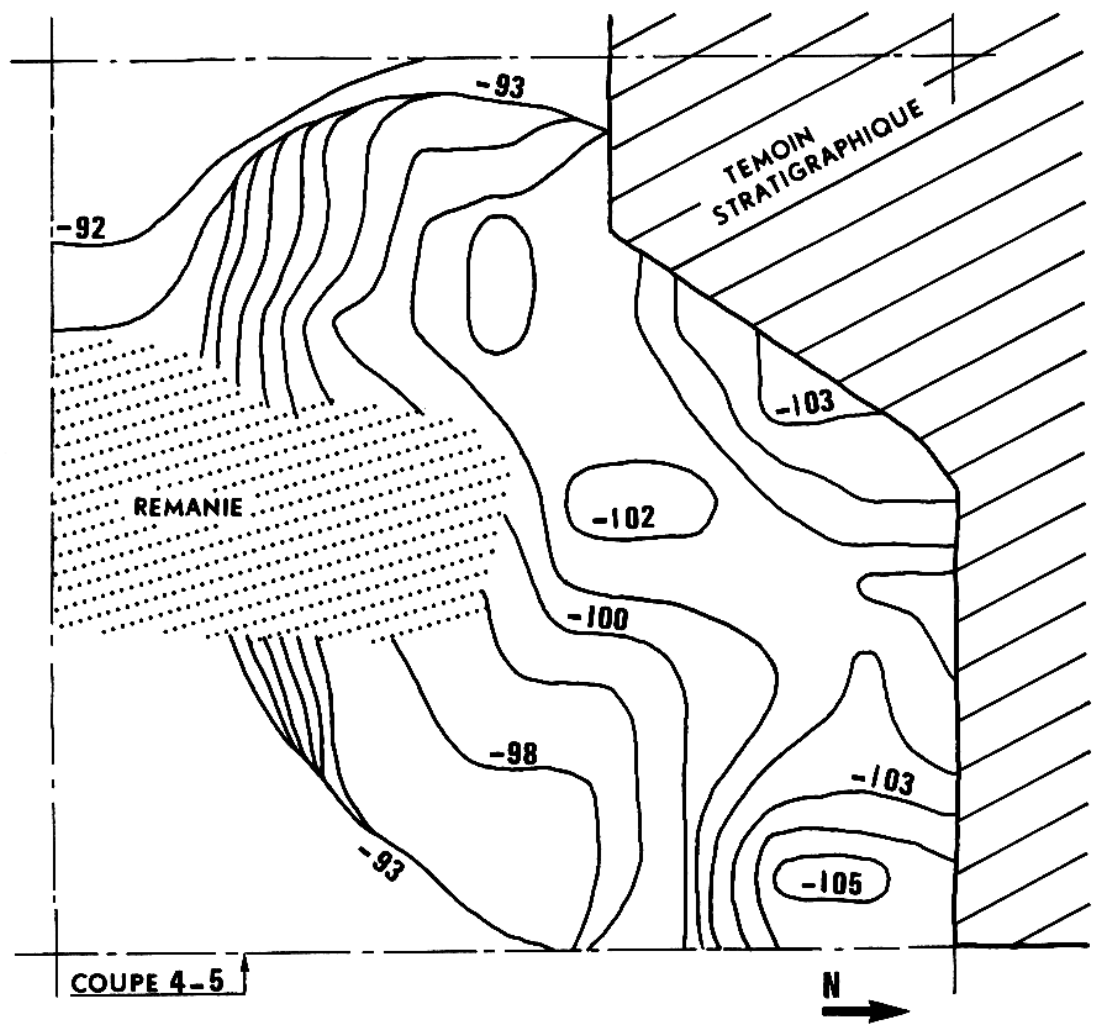

Fig. 4 - Topographie de la dépression dans laquelle le dépôt cendreux Gj est contenu.

succession Gb1-Gf1. Le second (Gb2) de $20 \mathrm{~cm}$ sur $15 \mathrm{~cm}$ de surface, constitué d'une même cendre blanche que Gb1, était disjoint de la première banquette. Quoique mal scellé sur son interface ( $G$ interstratifié) et donc légèrement déplacé, il appartient incontestablement à l'accumulation Gb1-Gf1.

Les documents lithiques en relation avec le débitage sont au nombre de 55. On dénombre 11 éclats entiers et 23 fragments, 2 lamelles entières dont une hypermicrolithique et 6 fragments lamellaires renforcés par 3 fragments de style lamellaire. Ces lamelles sont débitées dans le style de Coincy (Rozoy, 1968). Les Iongueurs des produits entiers sont comprises entre $3,5 \mathrm{~mm}$ et $32 \mathrm{~mm}$. Les débris sont pour l'essentiel des fragments thermiques ( 9 pour 10 ).

L'outillage est composé de quatre éléments. On distingue en $\mathrm{Gb1}$, une lamelle étroite à bord abattu dont le côté opposé est partiellement bordé (fig. $6, n^{\circ} 1$ ), une lamelle à coches multiples établie sur un support régulier à trois pans (fig. $6, n^{\circ} 2$ ), un débris retouché par un bordage partiel. Le lambeau Gb2 contenait un microlithe trapézoïdale cassé obtenu par troncatures semi-abruptes in- verses et retouches couvrantes directes. II s'agit d'une armature tranchante de Montclus (fig. $6, n^{\circ} 5$ ).

\section{- Couche G interstratifiée}

$G$ interstratifiée entre les structures Gb1-Gf1 et Gj offre l'opportunité de saisir une phase chronologique de l'épais dépôt $\mathrm{G}$.

Seuls 10 fragments de péricarpes de noisettes (détermination P. Marinval) renseignent sur l'environnement végétal.

Les débris de faune mammalienne n'ont pu être déterminés étant donné leur état très fragmentaire et leur présence d'os d'Amphibiens.

Le débitage est représenté par 138 éléments dont 49 débris dominés par les fragments thermiques (36). On distingue 10 éclats entiers et 48 fragments, 8 lamelles entières et 9 fragments lamellaires auxquels s'ajoutent 14 fragments de style lamellaire. Les produits entiers sont de petite dimension, leur longueur se distribue entre 3 et $21 \mathrm{~mm}$. Les lamelles sont débitées dans le style de Coincy (fig. 6, nos 14 à 16). Les exemplaires entiers ou raccourcis (10) combustion. On note, cependant, la montrent tous des talons très réduits.

L'outillage compte 7 armatures et 1 outil du fonds commun. II s'agit d'une lamelle à retouche continue. Les retouches, petites et directes, sont très régulières et surtout finement denticulées (fig. $6, n^{\circ} 13$ ). Parmi les armatures on distingue 3 géométriques hyperpygmées : un triangle de Montclus (fig. $6, n^{\circ} 7$ ), un triangle de Montclus apparenté (fig. $6, n^{\circ} 6$ ), un triangle scalène court retouché sur les trois côtés (fig. 6 , $n^{\circ} 8$ ). Un fragment de lamelle étroite à bord abattu tronquée s'inscrit dans l'esprit de cette courte série, notamment par la retouche de ses deux bords (fig. $6, n^{\circ} 9$ ). Les lamelles étroites à dos sont représentées par 2 lamelles bordées (fig. $6, n^{\text {os }} 10$ et 11 ) et une lamelle à bord abattu (fig. 6, $\mathrm{n}^{\circ}$ 12).

\section{- Dépôt Gj}

Les sédiments de Gj sont contenus dans une dépression ellipsoïdale qui couvre les trois quarts de la surface du carré F5 (fig. 4). Une bordure verticale et pentue en définit le contour. La poursuite des fouilles sur les carrés contigus devra assurer définitivement la présence d'un volume circonscrit, c'est-à-dire d'une cuvette creusée. Nous étudions ici un dépôt en relation avec une activité anthropique.

L'environnement arboré révélé par les cendres du sédiment (travaux J.-E. Brochier) apparaît dominé par les Angiospermes. La présence du Noisetier est attestée par 48 fragments de péricarpe de noisette (détermination P. Marinval).

Le spectre faunique comprend le cerf, des suinés (sanglier/porc indifférenciés), le blaireau, un oiseau indéterminé et des amphibiens.

Une coquille brûlée de Columbella rustica (détermination J. CataliottiValdina) doit probablement être considérée comme un élément de parure (fig. $7, n^{\circ} 74$ ). La détérioration de la base du test ne permet pas d'identifier la perforation.

\section{- Le déBItAge DU DÉPót GJ}

L'abondance des documents lithiques permet une première étude détaillée.

Le débitage est représenté par 1343 éléments (tabl. 1). Cet en- 


\begin{tabular}{|c|c|c|c|}
\hline & & $\mathbf{N b}$ & $\%$ \\
\hline \multirow{4}{*}{$\begin{array}{c}\text { lame/lamelle } \\
\mathrm{L} / \mathrm{l}>2\end{array}$} & entière & 40 & 2.97 \\
\hline & raccourcie & 23 & \begin{tabular}{|l|}
1.71 \\
\end{tabular} \\
\hline & corps & 23 & 1.71 \\
\hline & sans bulbe & 34 & 2.53 \\
\hline \multirow{3}{*}{$\begin{array}{c}\text { fragment de } \\
\text { style } \\
\text { lamellaire } \\
\mathrm{L} / \mathrm{l}<2\end{array}$} & proximal & 45 & 3.35 \\
\hline & mésial & 31 & 2.3 \\
\hline & distal & 33 & 2.45 \\
\hline \multicolumn{2}{|c|}{ éclat entier } & 194 & $\mathbf{1 4 . 4 4}$ \\
\hline \multirow{2}{*}{$\begin{array}{l}\text { fragment } \\
\text { d'éclat }\end{array}$} & proximal & 44 & \begin{tabular}{|l|}
3.27 \\
\end{tabular} \\
\hline & autre & 348 & 25.91 \\
\hline \multicolumn{2}{|c|}{ débris informe } & 110 & \begin{tabular}{|l|}
8.19 \\
\end{tabular} \\
\hline \multicolumn{2}{|c|}{ débris thermique } & 421 & 31.34 \\
\hline \multicolumn{2}{|c|}{ total } & 1343 & \\
\hline
\end{tabular}

\begin{tabular}{|c|c|c|}
\hline lame/lamelle & 120 & 8.93 \\
\hline éclat entier & 194 & 14.44 \\
\hline total fragments & 501 & 37.3 \\
\hline total débris & 531 & 39.53 \\
\hline total & 1343 & \multicolumn{1}{|l}{} \\
\hline
\end{tabular}

nucleus

Tabl. 1 - Débitage du dépôt Gj.

semble est marqué par deux tendances : une forte représentation des débris d'origine thermique (31,5\% de l'ensemble) et l'importance de l'indice global de fragmentation des produits de débitage $(71,5 \%$, hors débris thermiques et informes).

Les produits lamellaires sont représentés par 3 courtes lames en relation avec la gestion du débitage, 37 lamelles entières et 80 fragments. Le taux de supports allongés s'établit donc à $9 \%$ de l'ensemble des éléments du débitage. Cette proportion est un indice résiduel restreint sans doute éloigné du rendement normal de la production utile comme on peut en juger par la nature des supports d'outils qui, pour $94 \%$ d'entre eux sont lamellaires ou d'équivalence lamellaire. De plus, on peut tenir comme fiable la détermination des fragments proximaux de style lamellaire qui élargissent le taux précédent. Ils présentent toujours un très petit talon et parfois une morphologie frontale en ogive assez typique parmi les produits de débitage lamellaires entiers ou raccourcis.

Les produits lamellaires contenus en Gj ne sont pas représentatifs de la totalité des classes dimensionnelles de produits réalisables au cours du débitage lamellaire. On en juge par la présence d'une grande lame à crête et de deux entames naturelles, dont les dimensions nous renseignent sur le potentiel qu'on eu certain nucléus, pour produire de grands supports lamellaires utiles, au moment de la constitution de la couche Gj. Ces trois courtes lames attestent un déficit de représentation des lamelles entre les classes des 20 à $45 \mathrm{~mm}$ (fig. 5).

Le contenu lamellaire de Gj est alimenté par une production (fig. 7 , $n^{\circ} 68$ ) obtenue à partir de nucléus nettement diminués, comme le montre le format des lamelles outrepassées qui ont emporté la base de leur nucléus (fig. $7, n^{\circ} 73$ ). Ce débitage lamellaire qu'on peut qualifier de pygmée et d'hyperpygmée a cependant contribué, même pour les éléments inférieurs au centimètre, à fournir des supports aux microlithes comme l'attestent une pointe à dos (fig. $7, n^{\circ} 18$ ) et les géométriques triangulaires hyperpygmées (fig. $7, n^{\circ s} 2$ à 5). II apparaît donc nécessaire de s'intéresser aux produits inférieurs au centimètre que la plupart des études négligent de considérer.

Le style des produits lamellaires est celui qu'on observe classiquement durant le Sauveterrien. Les bords sont ici souvent parallèles ou convergents, bien plus rarement di-

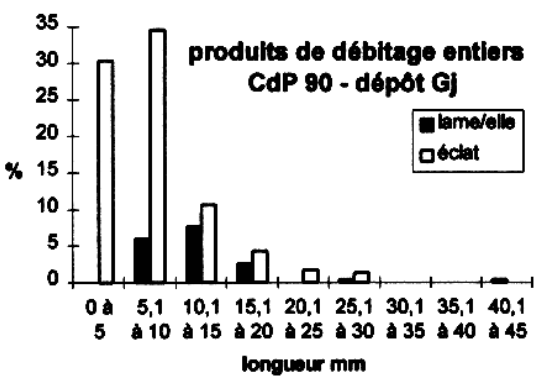

Fig. 5 - Représentation des produits de débitage entiers de Gj. Les deux courtes lames des classes 25.1 à 30 et 40.1 à 45 sont des crêtes.

vergents. Les arêtes dorsales fréquemment irrégulières et bifurquées déterminent le plus souvent une lamelle à deux pans ( 31 pour 40 lamelles entières).

Les talons sont très petits. Cette réduction de la surface induit une morphologie des talons lisses plutôt punctiforme ou linéaire qui justifie que nous les regroupions en un même ensemble qui compose les trois quarts des talons des lamelles entières (30 pour 40 ). Une telle tendance se retrouve sur les lamelles
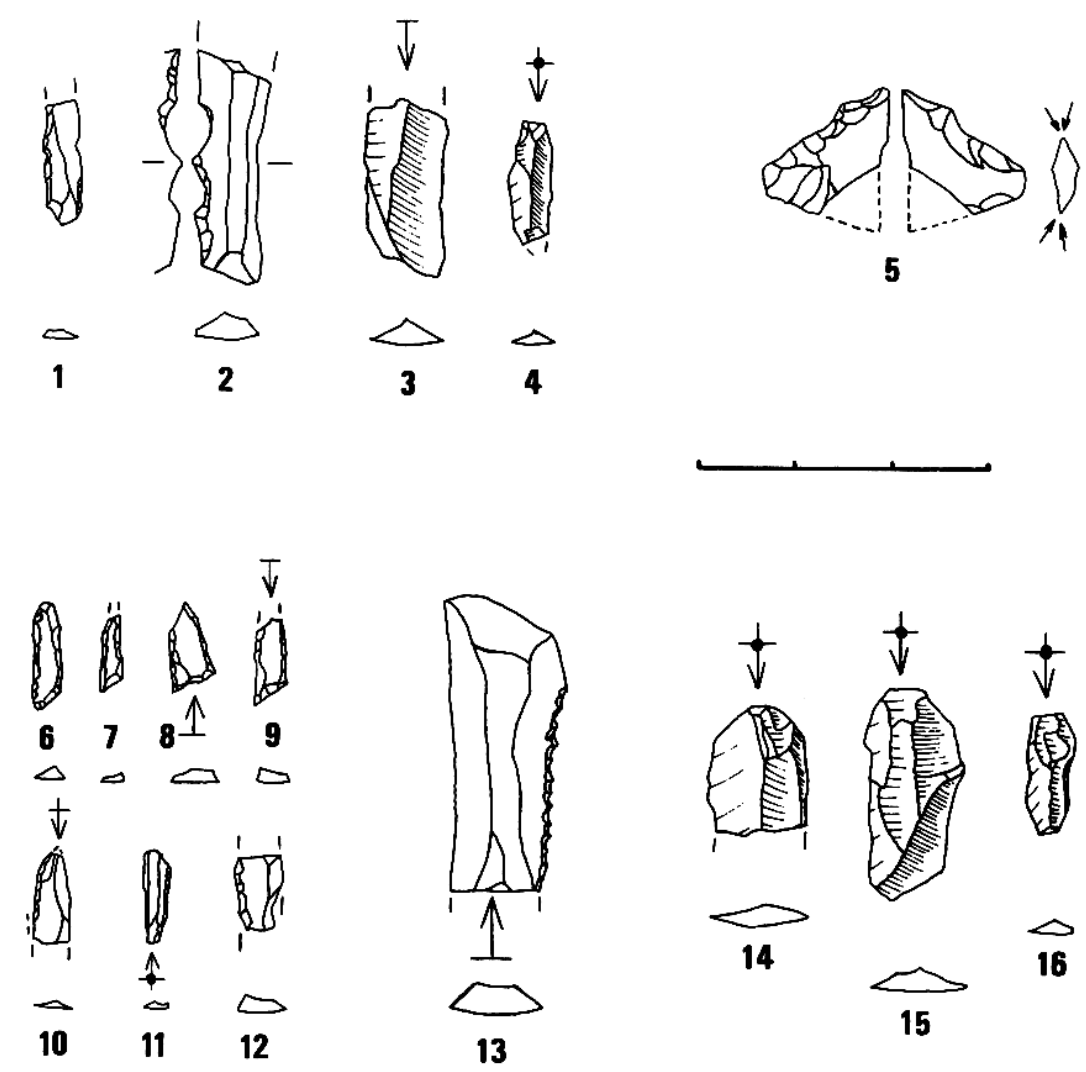

Fig. 6 - Industrie lithique. 1 à $4: G b 1 ; 5: G b 2 ; 6$ à $16: G$ interstratifiée. Gb1 - $1:$ lamelle étroite à dos; 2 : lamelle à coches multiples; 3 et 4 : lamelles brutes. Gb2 $-5:$ armature tranchante de Montclus. $G$ interstratifiée -6 à $8:$ triangles de Montclus et apparentés, 9 : lamelle étroite à dos tronquée; 10 et 11 : lamelles étroites bordées ; 13 : lamelle à retouches continues; 14 à 16 : lamelles brutes. 


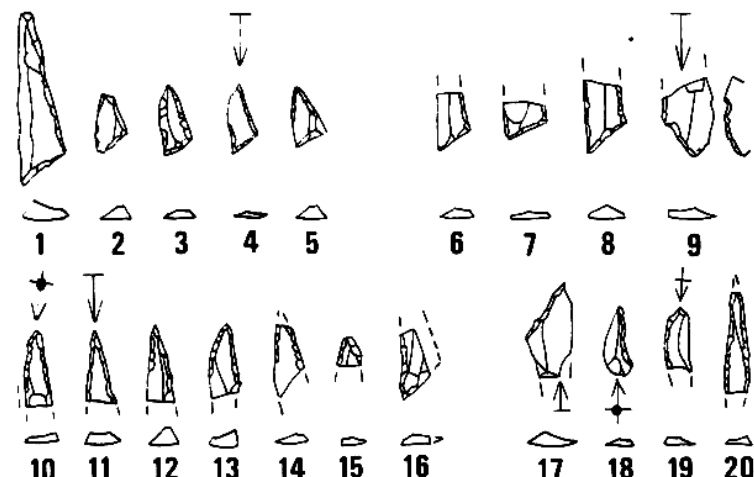

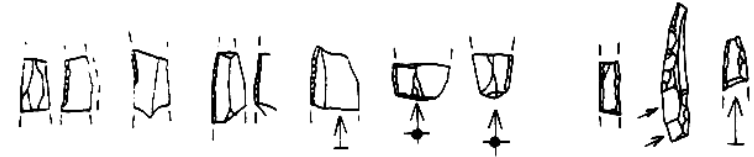

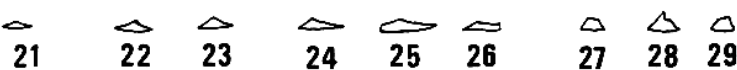

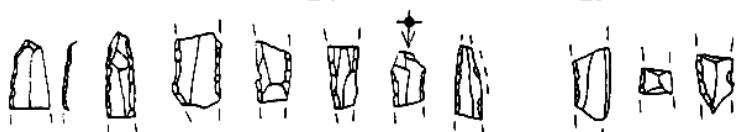

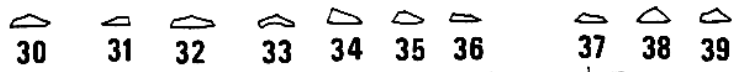

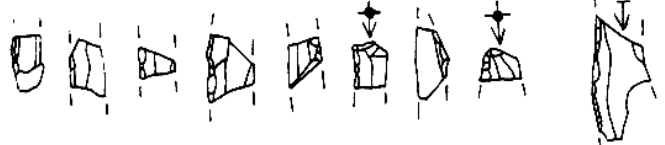

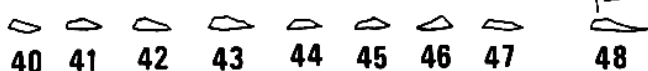
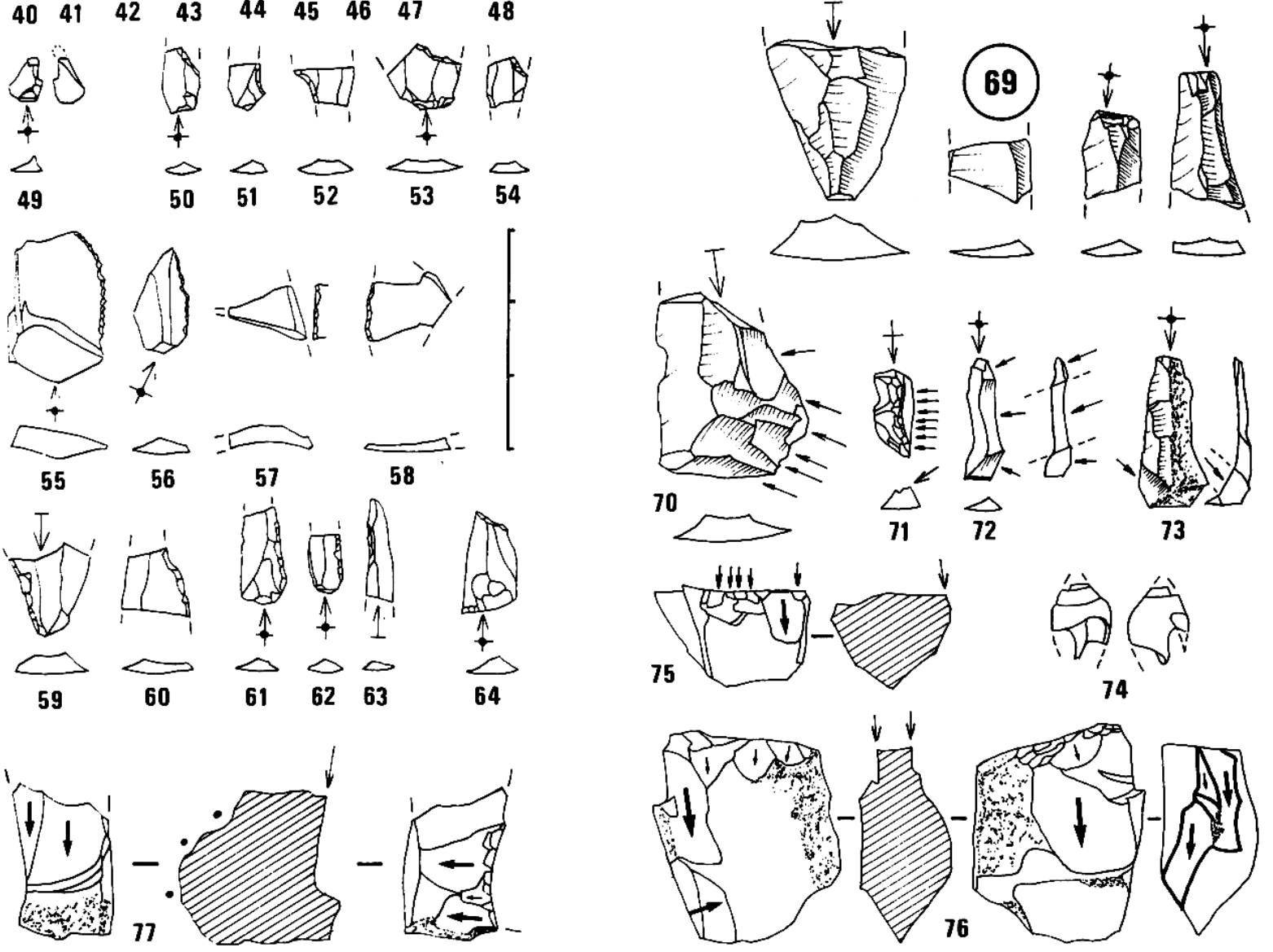

Fig. 7 - Industrie lithique et parure de Gj. 1 à $5:$ géométriques ; 6 à $9:$ lamelles étroites à dos tronquées ; 10 à $16:$ fragments apicaux ; 17 à 19 : pointes hyperpigmées ; 20 : pointe de Sauveterre; 21 à 26 : lamelles étroites bordées ; 27 à $29:$ débris et ébauche de pointes de Sauveterre; 30 à 33,35 et 36 : lamelles étroites à dos et bordage ; 34 : lamelle étroite à 2 dos; 37 à $47:$ lamelles étroites à dos; 48 : lamelle à dos ; $49:$ microburin ; 50 à 54 : lamelles cassées dans une encoche ; 55 à 58 : éclats retouchés; 59 à 63 : lamelles retouchées ; 64 : lamelle tronquée ; 65 à 67 : lames à crêtes ; 68 et 69 : produits de débitage ; $74:$ Columbella rustica ; 75 à $77:$ nucléus. 
raccourcies et sur de nombreux fragments proximaux de style lamellaire. Le restant comprend des talons facettés ou dièdres et des talons corticaux ou naturels.

La plupart des bulbes de percussion présentent des nodosités étalées et surtout diffuses ( 35 pour 40 ). Les petits bulbes localisés et bien marqués sont peu fréquents.

L'aménagement de la corniche est lisible sur la moitié des lamelles entières (22 pour 40 ). II se présente comme un micro-étagement ou un micro-chanfrein. Cet aménagement concerne également les lamelles hyperpygmées. II démontre, à ce stade d'exhaustion du nucléus, une préparation soignée des corniches, conférant aux micro-lamelles un statut de produits recherchés au moins partiellement.

Les éclats entiers et surtout leurs nombreux fragments composent une large part des documents lithiques $(14,44 \%$ et $29,18 \%)$. Ils confirment les mêmes tendances dimensionnelles que celles des lamelles. Une part prépondérante des éclats entiers montre des longueurs inférieures au centimètre (152 pour 194).

La nature des produits de débitage, notamment leurs dimensions, est appréhendable par la présence de trois nucléus résiduels (fig. 7 , $n^{\circ} 75$ à 77). Étant donné leur stade d'exhaustion, leur production devait se limiter à des petits éclats lamellaires ou à des micro-lamelles.

Un exemple d'organisation du débitage est illustré sur un nucléus par deux séquences d'extraction de direction croisée, établie chacune sur une face autonome (fig. $7, \mathrm{n}^{\circ} 77$ ). II est possible de préciser le mode d'exploitation unipolaire d'un autre nucléus. Le support originel est étroit et défini par des faces latérales naturelle et corticale. Les lamelles furent extraites dans l'épaisseur du support (extractions burinantes). Dans un second temps des éclats plus ou moins grands furent dégagés sur les deux flancs à partir du même plan de frappe jusqu'à ouvrir l'angle de chasse et conférer à ce nucléus l'aspect d'une pièce esquillée partielle (fig. $7, n^{\circ} 76$ ).

La prédétermination des débitages lamellaires contemporains de Gj a pu se concevoir directement à partir du dièdre d'un bloc brut comme le montrent deux crêtes na- turelles (fig. $7, n^{\circ s} 65$ et 67 ) ou à partir d'une préparation comme l'atteste une crête à un versant préparé (fig. $7, n^{\circ} 66$ ). Deux produits de débitage présentent sur leur face supérieure les négatifs de débitages croisés. II s'agit d'un flanc de nucléus (fig. $7, n^{\circ} 70$ ), et d'une lamelle secondaire de recoupe d'une préparation en crête (fig. $7, n^{\circ} 72$ ). On note également l'ablation, par un enlèvement orthogonal, d'une corniche de nucléus bien réduite (fig. $7, n^{\circ} 71$ ).

Les débris se composent de fragments informes sur lesquels aucune face d'éclatement n'est lisible. Trois de ces débris incluent une corniche partielle de nucléus.

Les débris thermiques sont majoritaires. Ce sont des fragments totalement transformés par des cupules thermiques et des éclats thermiques.

\section{- L'OUTILlage du déPÓt Gj}

Le dépôt $\mathrm{Gj}$, compte tenu du faible volume de sédiment fouillé, peut être considéré comme bien documenté malgré un nombre d'outils limité à 65 (tabl. 2).

Cette série se caractérise par la prépondérence des armatures avec 49 éléments et la suprématie des outils ouvrés sur des supports lamellaires (61 éléments). Les classes d'outils ne sont pas toutes représentées. Nous retrouvons cependant les très classiques groupes des éclats et des lamelles retouchées qui constituent le fonds commun (10 individus). Les pièces techniques sont bien représentées avec 6 éléments. Les armatures comptent pour plus de la moitié des lamelles étroites à dos. Les géométriques triangulaires représentent 1 armature sur 10 , que renforcent à peine les lamelles à dos tronquées (4 éléments). Le groupe des pointes à dos, étonnement varié, n'est représenté que par 4 exemplaires. On note l'importance des fragments apicaux indéterminables (8 débris).

\section{- Fonds commun}

Les éclats retouchés comprennent deux fragments informes modifiés par des retouches régulières, abruptes inverses (fig. $7, n^{\circ} 57$ ) et semi-abruptes directes (fig. $7, n^{\circ} 58$ ) proches de bordages. Deux éclats entiers sont transformés en continu sur un bord unique par une petite retouche directe, très régulière et den- ticulante (fig. $7, \mathrm{n}^{\text {os }} 55$ et 56 ), tout à fait comparable à celle qui modifie en continu une lamelle de la couche G interstratifiée (fig. 6, $n^{\circ} 13$ ).

Les lamelles sont retouchées en continu ou partiellement. Trois d'entre elles ont un format en rapport avec les microlithes et font douter de leur réelle appartenance au fonds commun (fig. $7, n^{\text {os }} 61$ à 63 ). Nous justifions leur classement par une retouche plate parallèle au pan du support sans transformation de son

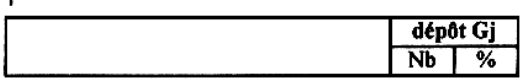

\begin{tabular}{|l|l|l|}
\hline 18 éclat mince retouché & 4 & 6.15 \\
\hline 19 éclat mince denticulé & & \\
\hline 20 éclat mince tronqué & & \\
\hline 21 éclat épais retouché & & \\
\hline 22 éclat épais denticulé & & \\
\hline 23 éclat épais tronqué & & \\
\hline
\end{tabular}

23 éclat épais tronqué

\begin{tabular}{|l|c|c|}
\hline 24 lamelle à retouches continues & 4 & 6.15 \\
\hline 25 lamelle à retouches partielles & 1 & 1.53 \\
\hline 26 lamelle à retouches alternes & & \\
\hline 28 lamelle à coches multiples & & \\
\hline 30 lamelle tronquée & 1 & 1.53 \\
\hline
\end{tabular}

\begin{tabular}{|c|c|c|}
\hline 27 lamelle à coche unique & & \\
\hline 29 lamelle cassée dans une encoche & 5 & 7.69 \\
\hline 00 microburin & 1 & 1.53 \\
\hline
\end{tabular}

\begin{tabular}{|l|l|l|}
\hline 31 pointe à troncature trés oblique & & \\
\hline 32 pointe à dos rectiligne & & \\
\hline 33 pointe à dos courbe & & \\
\hline 34 pointe de Sauveterre & 1 & 1.53 \\
\hline
\end{tabular}

\begin{tabular}{|l|l|l|}
\hline $\begin{array}{l}35 \text { micro-pointe à troncature trés } \\
\text { oblique }\end{array}$ & 1 & 1.53 \\
\hline 36 micro-pointe à dos rectiligne & 1 & 1.53 \\
\hline 37 micro-pointe à dos courbe & 1 & 1.53 \\
\hline 38 micro-pointe de Sauveterre & & \\
\hline
\end{tabular}

\begin{tabular}{|l|c|c|}
\hline 47 lamelle étroite à bord abattu & & \\
\hline 48 frag. de lamelle étroite à bord abattu & 27 & 41.5 \\
\hline 51 lamelle à bord abattu & & \\
\hline 52 frag de lamelle à bord abattu & 1 & 1.53 \\
\hline
\end{tabular}

52 frag. de lamelle à bord abattu

\begin{tabular}{|l|l|l|}
\hline 56 triangle isocèle & & \\
\hline 57 triangle scalène ordinaire court & 3 & 4.61 \\
\hline 58 triangle scalène ordinaire long & & \\
\hline 59 triangle scalène allongé & & \\
\hline 60 triangle scalène ordinaire élancé & 1 & 1.53 \\
\hline 61 triangle scalène allongé élancé & 1 & 1.53 \\
\hline 62 triangle scalène éffilé & & \\
\hline 63 triangle scalène effilé allongé & & \\
\hline 64 triangle de Montclus & & \\
\hline 65 triangle de Montclus apparenté & & \\
\hline
\end{tabular}

65 triangle de Montclus apparenté

\begin{tabular}{|l|c|c|}
\hline $\begin{array}{l}\text { 49 lamelle étroite à bord abattu } \\
\text { tronquée }\end{array}$ & & \\
\hline 50 frag. lamelle étr.à bord ab. tronquée & 3 & 4.61 \\
\hline 53 lamelle à bord abattu tronquée & & \\
\hline 54 frag. lamelle à bord abattu tronquée & 1 & 1.53 \\
\hline
\end{tabular}

\begin{tabular}{|l|l|l|}
\hline 66 partie apicale indéterminable & 8 & 12.3 \\
\hline
\end{tabular}

\begin{tabular}{|l|c|c|}
\hline total fonds commun & 10 & 15.4 \\
\hline total pièces techniques & 6 & 9.23 \\
\hline total armatures & 49 & 75.4 \\
\hline
\end{tabular}

\begin{tabular}{|l|l|l|}
\hline total outillage & 65 & \\
\hline
\end{tabular}

aménagements de la liste : 1- création d'une classe de pièces techniques et intégration des microburins. 2- création d'une classe indépendante de lamelles à dos tronquées, intégrée après celle des triangles.

Tabl. 2 - Liste type partielle du mésolithique sauveterrien d'après Valdeyron 1994. 
contour. Deux fragments semblent bien caractéristiques de cette classe d'outil (fig. $7, \mathrm{n}^{\text {os }} 59$ et 60 ). Une lamelle est transformée par une troncature oblique distale (fig. $7, n^{\circ} 64$ ).

\section{- Pièces techniques}

Elles se répartissent entre 5 lamelles cassées dans une encoche (fig. $7, n^{\text {os }} 50$ à 54 ) et un microburin proximal (fig. $7, n^{\circ} 49$ ).

\section{- Armatures}

Toutes les lamelles à dos sont des fragments. On dénombre 27 lamelles étroites et 1 lamelle large. La retouche, bien sûr abrupte, peut se limiter à un bordage seul comme on l'observe sur 6 lamelles (fig. 7, $n^{\text {os }} 21$ à 26). Ce bordage est une fois inverse (fig. $7, \mathrm{n}^{\circ} 21$ ) et une fois alterne (fig. $7, n^{\circ} 23$ ). Les côtés abattus des autres lamelles sont 10 fois bilatéraux. On observe alors l'association fréquente entre une retouche abrupte d'un côté et un bordage de l'autre (6 cas), parfois alterne (fig. 7, $n^{\circ} 30$ ). On note donc l'importance générale du bordage dans le façonnage des lamelles à dos. Nous attribuons trois fragments de lamelles à deux bords abattus à de probables débris de pointes de Sauveterre (fig. 7, nos 27 à 29). Dans un cas il peut s'agir d'une ébauche (fig. 7 , $n^{\circ} 28$ ).

Parmi les pointes à base non retouchée, on discerne une pointe de Sauveterre (fig. $7, n^{\circ} 20$ ), une micropointe à dos rectiligne (fig. $7, n^{\circ} 18$ ), une micro-pointe à dos courbe (fig. $7, n^{\circ} 19$ ) ainsi qu'une micropointe à troncature très oblique (fig. $7, n^{\circ} 17$ ).

Les géométriques sont représentés par 5 triangles scalènes. On distingue un grand scalène allongé élancé et pygmée (fig. $7, n^{\circ} 1$ ) et surtout 4 scalènes ordinaires parmi lesquels 1 élancé (fig. $7, n^{\circ} 3$ ). Leur dimension les situent très en dessous du seuil des hyperpygmées $(10 \mathrm{~mm})$, c'est-à-dire entre 6 et $7 \mathrm{~mm}$. Le grand côté de ces hypermicrolithes est transformé pour trois d'entre eux par un bordage partiel (fig. $7, n^{\circ} 4$ et 5) ou un bord abattu partiel (fig. 7 , $\mathrm{n}^{\circ} 3$ ).

Quatre lamelles à bord abattu tronquées, 3 étroites et 1 large, sont toutes des fragments et certaines de probables débris de triangle scalène (fig. 7, comparer $n^{\circ s} 1$ et 6). On note la retouche des deux bords sur deux d'entre elles (fig. $7, n^{\circ s} 7$ et 8 ) et un bord ébréché inverse, alterne au bord abattu sur la lamelle large (fig. $7, n^{\circ} 9$ ).

Huit débris pointus sont des fragments apicaux. Ils pourraient morphologiquement constituer des débris de géométriques triangulaires (fig. $7, \mathrm{n}^{\text {os }} 10$ à 16).

\section{- NOUS RESUMONS LES fAITS CONCER- NANT LE DEPOT Gj COMME SUIT :}

- Dépôt sédimentaire localisé, contenu dans une dépression du sol.

\section{- Fond de la dépression rubéfié.}

- Sédiment riche en cendre de bois (travaux J.-E. Brochier).

- Dépôts en plusieurs étapes. Interstratifications dans la masse du sédiment : de langues de débordement grisâtres issues du sol encaissant $(G)$; de passées orangées formant des feuillets indurés et rubéfiés.

- Forte représentation des débris lithiques thermiques.

- Produits de débitage entiers composés en très grande majorité d'éclats minuscules de moins de $1 \mathrm{~cm}$ et de moins de $5 \mathrm{~mm}$.

- Absence de certaines classes dimensionnelles parmi les produits de débitages.

- Équilibre typologique des armatures marqués par la suprématie des lamelles à dos et la discrétion des géométriques triangulaires.

- Faune très fragmentée et brûlée.

\section{LES RESTES OSSEUX (A.B.)}

Les ossements des couches Gb1, Gf1, Gb2, G interstratifiée et Gj qui ont fait l'objet de cette étude présentent globalement un aspect homogène.
On peut estimer que $90 \%$ des restes sont brûlés (carbonisés et calcinés), une partie d'entre eux présentant des concrétions cendreuses. La grande majorité des fragments mesure entre 0,5 et $1,5 \mathrm{~cm}$, la longueur maximale n'exédant pas $4 \mathrm{~cm}$. Proportionnellement, les restes dentaires sont très minoritaires, pour l'essentiel des fragments d'émail, ce qui pourrait refléter une caractéristique du dépôt initial plutôt que le résultat d'un processus taphonomique naturel.

Ces caractéristiques rendent la détermination spécifique très ardue, de telle sorte que seules 16 pièces ont été identifiées :

La couche Gj est la mieux documentée (tabl. 3), tant en nombre de restes déterminés (15) qu'en poids total de reste $(200 \mathrm{~g}$, soit plus de la moitié du poids total de la faune provenant des niveaux ci-dessus mentionnés). Cerf, suinés (Sanglier ou Porc) et Blaireau y sont représentés. La présence d'oiseau indéterminé et d'amphibiens est attestée par des os brûlés. Un fragment de bois présentant de fortes perlures pourrait être attribué à du chevreuil. Les deux petits fragments de bois de cerf représentés dans ce niveau sont calcinés.

La composition de la couche $\mathrm{Gj}$ présente des analogies avec les faunes mésolithiques connues par ailleurs : Cerf et Suinés sont les principales espèces d'ongulés, le Blaireau, fréquemment attesté dans les faunes de cette période, il est présent notamment dans le Sauveterrien de Rouffignac (Delpech et Suire, 1974), est cependant ici la seule espèce de petit carnivore (on peut noter l'absence de la Martre, généralement plus fréquente encore).

Les comparaisons fondées sur des échantillons de petite taille étant risquées, les remarques suivantes sont présentées à titre d'hypothèses :

\begin{tabular}{|c|c|c|c|c|}
\hline \multicolumn{2}{|l|}{ Espèces } & \multirow{2}{*}{ Gb1 } & \multirow{2}{*}{$\frac{\mathrm{Gj}}{3}$} & \multirow{2}{*}{$\begin{array}{c}\text { Total } \\
\mathbf{3}\end{array}$} \\
\hline Cervus elaphus & Cerf & & & \\
\hline & Cerf (bois) & 1 & 2 & 3 \\
\hline cf Capreolus capreolus & cf Chevreuil (bois) & & 1 & 1 \\
\hline Suinae & Sanglier / Porc & & 5 & 5 \\
\hline Meles meles & Blaireau & & 3 & 3 \\
\hline Aves & Oiseau indéterminé & & 1 & $\mathbf{1}$ \\
\hline Total & & 1 & 15 & 16 \\
\hline
\end{tabular}

Tabl. 3 - Décompte des restes déterminés par couche. Présence d'os d'amphibiens dans les couches $\mathrm{G}$ interstratifiée et $\mathrm{G}$. 
- Les caractéristiques extrinsèques du matériel évoque un dépôt lié à la présence de structure(s) de combustion ou de vidange, ce qui s'accorderait avec les observations de J.-E. Brochier. On peut alors se demander dans quelle mesure les spectres fauniques sont représentatifs à l'échelle de l'ensemble du site.

- Le cortège de faune issu de ces différents niveaux évoque un milieu boisé avec des zones ouvertes. II est intéressant de noter que dans le niveau $X$ de Roquemissou attribué à un Épipaléolithique d'après les caractéristiques typologiques (Bobœuf, 1991), le cheval est (encore) représenté, associé au cerf et au sanglier (Fontan, 1991).

\section{口ISCUSSION}

L'attribution chrono culturelle du foyer Gb1-Gf1 serait le Néolithique ancien. Outre l'armature tranchante de Montclus et la lamelle à coches multiples, des éléments d'appréciation indirecte nous confortent dans cette interprétation. L'éboulement des coupes issues des anciens travaux jusqu'à leur profil de stabilisation, a livré, après tamisage des sédiments mélangés, des documents lithiques et céramiques. Les premiers, une pointe du Martinet, un trapèze symétrique et une lamelle à coches multiples, sont rapportables, en l'état des connaissances régionales, à une phase récente du Mésolithique ou/et au Néolithique ancien caussenard. Le trapèze symétrique était encore enrobé de sédiment cendreux blanc. Les seconds, de qualité et d'aspect variés, sont difficiles à attribuer en l'absence de forme et de décors. Quatre petits tessons, dont un bord arrondi et étroit interrompant une panse qui s'élargit rapidement vers le fond du vase et un typique exemple de montage au colombin (gouttière), ont retenu notre attention car également encroutés de sédiments cendreux blanchâtre et grisâtre. Ces documents pourraient provenir du démantèlement de Gb1-Gf1 et pourraient conforter l'attribution de cette structure au Néolithique ancien.

Nous retenons donc l'hypothèse d'un horizon du Néolithique ancien qui comporterait au moins une structure (Gb1-Gf1) et occuperait la partie supérieure de la couche $\mathrm{G}$.

La présence d'un microlithe "sauveterrien", une lamelle étroite à dos, associé à une armature tranchante de Montclus dans le foyer Gb1-Gf1 pose à nouveau la question de la pertinence des associations observées. II aurait été profitable de savoir si le foyer Gb1-Gf1 avait été établi dans une cuvette creusée, dès lors que son substrat ( $G$ interstratifié) peut être attribué au Mésolithique moyen montclusien. La question reste ouverte.

L'attribution des éléments lithiques de $G$ interstratifiée ne pose guerre de problème, à la seule restriction que cette faible série soit vraiment représentative. Les meilleures comparaisons qualitatives se réalisent avec les couches c4 et c3 du gisement héraultais de l'Abeurador (Vaquer et Barbaza, 1987), les couches éponymes 22 à 17 du site gardois de la baume de Montclus (Rozoy, 1978) et localement les ensembles VIII à IV du locus 1 de Roquemissou (Bobœuf, 1995) qui caractérisent une période avancée de la phase montclusienne du Sauveterrien.

Le spectre documentaire de la série lithique du dépôt Gj pose la question de sa signification face aux séries que fournissent les gisements sauveterriens jusqu'alors étudiés. Ces séries proviennent de sol(s) ou de niveaux généraux d'occupation(s) dans lesquels sont associés les témoins d'activités variées qui, cumulés, conduisent à la pondération certaine des spectres documentaires. Classiquement les études du lithique sauveterrien analysent ce type d'échantillonnage, ce qui paraît légitime dès lors qu'il s'agit d'en caractériser le faciès culturel ou le degrés d'évolution chronologique (Rozoy, 1978 ; Barbaza et alii, 1991 ; Valdeyron, 1994), ce qui limite dans notre cas précis les possibilités de comparaison.

La nature de Gj semble liée à un faciès de dépôt. L'activité indéterminée (ou les activités?) a induit une sélection parmi les documents lithiques et probablement parmi l'ensemble des restes, notamment fauniques dont on peut souligné l'extrême fragmentation et la combustion intense. Elle se traduirait par un déficit dans la représentation des produits de débitage et par un équilibre original de la représentation typologique des armatures. L'industrie de Gj pourrait correspondre à l'équilibre normal d'un faciès d'activité.

L'absence d'une partie de la production lamellaire est notable puisqu'il s'agit des classes des 20 à $45 \mathrm{~mm}$ qui nourrissent habituellement en supports le groupe des lamelles retouchées et les plus grands microlithes. II n'est pas concevable d'évoquer une pénurie liée à l'économie de la matière première pour justifier le caractère dimensionnel de la série de $\mathrm{Gj}$. Les rares grands fragments (fig. $7, n^{\circ} 69$ ) et les crêtes (fig. $7, n^{\text {os }} 65$ à 67) en attestent l'existence pendant la formation des dépôts de Gj.

L'outillage présente également des originalités : équilibre des types d'armatures, large suprématie des lamelles à dos face aux géométriques triangulaires, cependant partiellement pondérable par les fragments apicaux et certaines lamelles à dos tronquées. Si on se fie à la morphologie des seuls triangles entiers on plaiderait pour un calage chronologique du dépôt excluant la phase moyenne ou montclusienne du Sauveterrien. On ne peut négliger une datation récente pour le Sauveterrien de Gj si on prend en compte le caractère dimensionnel des armatures, notamment des géométriques et des micro-pointes qui affirment une nette tendance à l'hypermicrolithisme ainsi que la retouche partielle du troisième côté observée sur trois triangles. Les fragments apicaux, systématiquement retouchés sur les deux côtés, renvoient également à une ambiance montclusienne. II parait sage de ne pas trancher prématurément. La présence de minuscules géométriques scalènes hyperpygmées et les premières occurrences de retouches partielles et même totales du troisième côté apparaissent très tôt, dans des contextes sauveterriens antérieurs à 9140 BP $(9140 \pm 160$ BP, LY 4448, c5b), notamment en Quercy dans les couches $c 5 c$ et $c 5 d$ de Fontfaurès (Barbaza et alii, 1991), ainsi qu'au pied de la Montagne Noire (Hérault) où ces phénomènes sont patents avant $8740 \mathrm{BP}(8740 \pm 90 \mathrm{BP}$, MC2144, c5) dans la couche c6 de la Balma de l'Abeurador (Vaquer et Barbaza, 1987).

Nous retenons que les particularités dimensionnelles des produits de débitage, l'équilibre de représentation des types d'armatures et éventuellement les singularités typologiques des géométriques sont ici induits par des facteurs sélectifs à mettre en relation avec la nature du dépôt, c'est-à-dire avec l'activité ou les activités dont il est l'expression résiduelle. 
Ces observations doivent être consolidées par l'élargissement du spectre documentaire du "sol" avec lequel $\mathrm{Gj}$ entretient des relations de contemporaneiité.

La coquille de Columbella rustica, mollusque marin vivant sur le littoral méditerranéen, intègre les Mésolithiques responsables de $\mathrm{Gj}$ dans le courant symbolique général qui semble prévaloir durant le Sauveterrien. Assez pauvres en parures, les dépôts sauveterriens livrent préférentiellement des coquilles de columbella dont on peut noter la vaste répartition géographique et la diffusion jusqu'en milieu continental (la Fru, Savoie). Leur découverte pourrait être plus fréquemment attachée à la phase moyenne ou montclusienne du Sauveterrien et à sa phase récente à trapèzes, comme à Montclus c22-19 et c18-15 dans le Gard (Rozoy, 1978), au Pey de Durance c2 dans les Bouches-du-Rhône (Valdeyron, 1994), à la grotte Lombard dans les Alpes-Maritimes (Binder et alii, 1991), à la Fru aire III c2 et c3 en Savoie (Pion et alii, 1990) et au Pas de la Charmate $\mathrm{c} 2 \mathrm{a}$ et $\mathrm{c} 1$ en Isère (Bintz, 1995). Notons toutefois que les gisements sauveterriens moyen sont aussi les plus nombreux.

Les éléments botaniques que livrent les premières analyses géo-archéologiques et carpologiques ne conduisent pas à caractériser précisément le cadre bio-chronologique des dépôts de l'ensemble gris $G$ (Gb1-Gf1, G interstratifiée, Gj). Cette séquence, comme l'indique la faune, montrerait un environnement boisé coupé d'espace dégagé, où prospèrent les Angiospermes parmi lesquels le Noisetier est rigoureusement attesté. De telles conditions biologiques sont en place dans le milieu montagnard de l'Aubrac, distant de $30 \mathrm{~km}$, dès la fin du Préboréal, au cours du Boréal et de l'Atlantique (Beaulieu et alii, 1985). II y a tout lieu de penser que le milieu caussenard, plus clément, a favorisé la mise en place plus précoce de la flore thermophile.

\section{PERSPECTIVES}

La baume du Clos de Poujol offre un potentiel d'étude diachronique des comportements mésolithiques et néolithiques ancien en milieu caussenard.

L'ensemble rouge $(R)$ devra être documenté. La série issue des fouilles de P.-M. Blanquet montre que des occupations historique (céramiques tournées) et néolithique sont envisageables pour la partie supérieure du remplissage.

La partie inférieure ou ensemble gris $(G)$ permettra de préciser les comportements d'acquisition et de consommation des ressources animale, végétale et minérale, dans le cadre du passage des activités prédatrices à la production de la biomasse animale et végétale, ce que rend possible la présence d'horizons mésolithiques et néolithique ancien.

On abordera la question de la signification des aspects de la variabilité des industries sauveterriennes, non seulement à l'intérieur du territoire d'acquisition et selon les modalités sitologiques, ce qui reste un objectif prioritaire (Barbaza et alii, 1991, p. 251-252), mais au sein du site même où l'association entre structures patentes ou latentes et industrie lithique devrait amener à clarifier la variabilité des assemblages lithiques et à la rendre intelligible notamment dans le cas de site de spécialisation.

Les observations réalisées sur l'industrie contenue en Gj présenteraient moins d'intérêts si elles n'étaient pas corrélables précisément à un contexte de dépôt avec lequel elle entretient des liens d'intimité qui permettent d'introduire la notion de faciès d'activité dans sa définition. La prise en compte de l'étude des industries et des documents naturalistes dans le détail de leur contexte de dépôt, c'est-à-dire les structures ou les épandages de sédiment distinct, doit favoriser la compréhension des comportements mésolithiques sauveterriens.

\section{Bibliographie}

BARBAZA M. et alii (1991) - Fontfaurès en Quercy. Archives d'Ecologie Préhistorique $n^{\circ} 11$. École des Hautes Etudes en Sciences Sociales, 1991, 270 p.

Beaulieu J.-L. de, Pons A. et Reille M. (1985) - "Recherches pollenanalytiques sur l'histoire tardiglaciaire et holocène de la végétation des monts d'Aubrac (Massif Central, France)." Review of paléobotany and palynology, Elsevier Science Publishers B.V., Amsterdam, 44, p. 37-80, 11 fig., 2 tabl.

BINDER D. et alii (1991) - Une économie de chasse au Néolithique ancien. Monographie du C.R.A. $n^{\circ} 5$, Editions du C.N.R.S., 243 p., 92 fig., 40 tabl.
BINTZ P. (1995) - "Abri mésolithique du Pas de la Charmate. Chatelus (Isère)". Livret-guide de l'excursion Préhistoire et Quaternaire en Vercors. $V^{\circ}$ Congrès International U.I.S.P.P. $\mathrm{XI}{ }^{\circ}$ commission. Épipaléolithiques et Mésolithique en Europe. Grenoble, France, 18 au 23 septembre 1995 . p. 104-117, 11 fig.

BOBCEUF M. (1991) - "Remarques préliminaires sur les industries lithiques du gisement de Roquemissou (Aveyron)". In : Mésolithique et néolithisation en France et dans les régions limitrophes. Actes du $113^{\circ}$ Congrès National des Sociétés Savantes, Strasbourg, 5-9 Avril 1988, Éditions du CTHS, p. 91-98, 4 fig.

BOBCEUF M. (1995) - "Sauveterrien et Roucadourien. Les outillages lithiques du Locus 1 de l'abri-sous-roche de roquemissou". Bulletin de la Société Préhistorique Française, p. 54-69, 22 fig., 3 tabl.

Delpech F. et Suire C. (1974) - "La faune mésolithique et post-mésolithique du gisement de Rouffignac". In : Barrière C., Rouffignac. L'Archéologie (2 partie). Université de Toulouse le Mirail. Travaux de I'Institut d'Art Préhistorique. t. XVI, p. 49-93.

FONTAN P. (1991). - "Étude préliminaire de la faune du gisement de Roquemissou (Aveyron)". In : Mésolithique et néolithisation en France et dans les régions limitrophes. Actes du $113^{\circ}$ Congrès National des Sociétés Savantes, Strasbourg, 5-9 Avril 1988, éditions du C.T.H.S., p. $87-90$ 2 tabl.

PION G. et alii (1990) - "L'abri de la Fru à Saint-Christophe (Savoie)". Gallia Préhistoire, tome 32,1990 , p. $65-123$, 54 fig., 15 tabl.

Rozoy J.-G. (1968) - "L'étude du matériel brut et des microburins dans l'Épipaléolithique (Mésolithique) francobelge". Bulletin de la Société Préhistorique Française, p. 365-390, 14 fig., 1 tabl.

Rozoy J.-G. (1978) - Les derniers chasseurs. L'Epipaléolithique en France et en Belgique, essai de synthèse. Bulletin de la Société Archéologique Champenoise, $\mathrm{N}^{\circ}$ spécial, 3 tomes, $1256 \mathrm{p}$., 294 fig., 259 pl., 80 tabl.

VALDEYRON N. (1994) - Le Sauveterrien. Culture et sociétés mésolithiques dans la France du Sud durant les $X^{\ominus}$ et $I X^{\ominus}$ millénaires $B P$. Thèse de doctorat, Université de Toulouse le Mirail, 584 p., 141 fig.

VAQUeR J. et Barbaza M. (1987) "Cueillette et Horticulture mésolithique : La Balma de l'Abeurador". In Premières communautés paysannes en Méditerranée occidentale. Actes du Colloque International du C.N.R.S. p. 231-242, 6 fig.

Marc BOBCEUF 34, rue Jean-Mermoz 92700 Colombes

Anne BRIDAULT U.R.A 1415 du C.N.R.S. Muséum National d'Histoire Naturelle 55 , rue Buffon 75005 Paris 